\title{
Influenza vaccination in asthmatic children: effects on quality of life and symptoms
}

\author{
H.J. Bueving*, J.C. van der Wouden*, H. Raat", R.M.D. Bernsen*, J.C. de Jongste ${ }^{*}$, \\ L.W.A. van Suijlekom-Smit ${ }^{+}$, A.D.M.E. Osterhaus ${ }^{\S}$, G.F. Rimmelzwaan ${ }^{\S}$, \\ M. Rutten-van Mölken ${ }^{f}$, S. Thomas*
}

Influenza vaccination in asthmatic children: effects on quality of life and symptoms. H.J. Bueving, J.C. van der Wouden, H. Raat, R.M.D. Bernsen, J.C. de Jongste, L.W.A. van Suijlekom-Smit, A.D.M.E. Osterhaus, G.F. Rimmelzwaan, M. Rutten-van Mölken, S. Thomas. C) ERS Journals Ltd 2004.

ABSTRACT: This study aimed to detect the effect of influenza vaccination on quality of life, symptomatology and spirometry in asthmatic children.

A randomised double-blind placebo-controlled trial in 696 (296 in 1999-2000 and 400 in 2000-2001) asthmatic children aged 6-18 yrs, which were vaccinated with either vaccine or placebo, was performed. Children participated for only one influenza season. They recorded symptoms in a diary and reported when symptom scores reached a predefined severity level. If this occurred research nurses visited them twice, first to take a pharyngeal swab and spirometry, and a week later to assess quality of life over the past illness week.

Compared with placebo, vaccination improved health-related quality of life in the weeks of illness related to influenza-positive swabs. However, no effect was found for respiratory symptoms recorded in the diaries during those weeks. Similarly, no differences were found for quality of life in all weeks of illness or for respiratory symptoms throughout the seasons.

Influenza vaccination was found to have a moderately beneficial effect on quality of life in influenza-positive weeks of illness in children with asthma.

Eur Respir J 2004; 24: 925-931.
*Dept of General Practice, ${ }^{\#}$ Dept of Public Health, "Pediatric Respiratory Medicine, ${ }^{+}$Dept of Pediatrics, ${ }^{\S}$ Dept of Virology, and ${ }^{f}$ Institute for Medical Technology Assessment, Erasmus MC - University Medical Center Rotterdam, The Netherlands.

Correspondence: J.C. van der Wouden, Dept of General Practice, room Ff305, Erasmus MC - University Medical Center Rotterdam, PO Box, 1738, The Netherlands.

Fax: 31104632127

E-mail: j.vanderwouden@erasmusmc.nl

Keywords: Asthma

influenza vaccination

quality of life

symptoms

Received: May 212004

Accepted after revision: June 122004

This study was supported by The Netherlands Organisation for Health Research and Development (ZonMw), The Hague, the Netherlands.
Influenza vaccination in children with asthma is recommended in most of Europe and North America [1]. Surprisingly, there are no randomised controlled trials confirming that vaccination is beneficial in this group [2]. The current authors recently reported the results of influenza vaccination in asthmatic children and did not find an effect on the number of influenza-related asthma exacerbations, or on their duration or severity; however, vaccination was found to shorten the duration of all asthma exacerbations by 0.8 days, irrespective of their cause [3].

Asthma exacerbations provide information about the status of the pulmonary system but do not capture the functional impairments (physical, emotional, and social) that are important in childrens' everyday lives [4]. Apart from preventing exacerbations, improvement in the well-being of asthmatic children should also be an important goal of the vaccine treatment. Influenza season has significant adverse effect on the quality of life (QoL) of school-age children and their families [5]. Influenza vaccine, by inducing a protection against influenza infection and subsequent illness, will reduce systemic and respiratory illness. It will also diminish related morbidity, such as missed schooldays, physician visits and the use of medication. This obviously will affect certain domains of QoL, e.g. symptoms, activity limitations and emotions.

This study reports whether influenza vaccination has an effect on childrens' asthma-related QoL during weeks of illness, on respiratory symptoms and on spirometric parameters in the corresponding periods, and on all symptoms throughout the seasons. To verify that the results are not confounded by differences in viral infections between groups, the authors also report on the viruses that were detected.

\section{Methods}

A randomised double-blind placebo-controlled parallel trial in children with asthma recruited through general practitioners (GP)s in Rotterdam, The Netherlands, and its surroundings during two influenza seasons, 1999-2000 and 2000-2001, was carried out. The design, which has been described in detail elsewhere [3], is briefly summarised here.

\section{Participants}

Children aged 6-18 yrs were included if they had used maintenance therapy for asthma or $>52$ doses of relief medication during the previous 12 months. Children could participate for only one season. Exclusion criteria were other chronic diseases, allergy to chicken protein and insufficient understanding of the Dutch language. The Medical Ethical 
Committee of Erasmus MC - University Medical Center Rotterdam approved the study.

\section{Intervention}

In both seasons, research nurses parenterally vaccinated all participants between 25 October and 24 November with either inactivated influenza vaccine (Solvay, Weesp, the Netherlands) or placebo. The vaccine composition for 1999-2000 was a combination of A/Sydney/5/97 H3N2-like, A/ Beijing/262/95 H1N1-like and B/Beijing/184/93-like strains and for 2000-2001 A/Moscow/10/99 H3N2-like, A/New Caledonia/ 20/99 H1N1-like and B/Beijing/184/93-like strains, as advised by the World Health Organisation. The placebo consisted of a buffered phosphate solution with the same $\mathrm{pH}$ value and was similar in appearance to the inactivated influenza vaccine.

\section{Objectives}

The study aimed to answer whether influenza vaccination in children with asthma has an effect on asthma-related QoL, respiratory symptoms and spirometric parameters during weeks of illness and on their symptomatology throughout the influenza season.

\section{Quality of life}

Health-related QoL was assessed by means of the Dutch version of the 23-item Paediatric Asthma Quality of Life Questionnaire (PAQLQ), which the current authors recently validated $[6,7]$. The PAQLQ consists of the domains symptoms (10 items), activities (five items) and emotions (eight items). The instrument measures well-being over a previous period of 7 days, a period that covers the usual period of symptoms and signs typically due to influenza. The score for every item ranges from 1-7, with higher scores indicating a better QoL.

When children reached the threshold of the severity level for symptoms a PAQLQ was administered 1 week later. In case of multiple weeks of illness the authors calculated the mean PAQLQ values. For all children, a baseline PAQLQ was administered when upper respiratory tract (URT) infection and lower respiratory tract (LRT) infection symptom scores had been beneath the threshold of four points for a period of at least 7 days. The authors considered a change between the total scores or domain scores of baseline versus illness of at least 0.5 points as a minimal important difference (MID). The clinical relevance of this difference has been assessed previously [6].

\section{Symptom scores and weeks of illness}

Participants (or their parents) were asked to fill in a diary, starting the day after vaccination, and daily score symptoms of URT and LRT symptoms. URT symptoms included sneezing, runny or stuffy nose, burning or watery eyes, sore throat, hoarseness, fever or shivering, headache and myalgia. LRT symptoms (asthma symptoms) included cough and wheeze during day and night and difficult breathing or shortness of breath. If present, respiratory tract symptoms were scored from one (mild) to three (severe) and summed; not fit to go to school or work because of symptoms was scored as two. The authors instructed participants to telephone the investigators when the summated scores for either URT or LRT symptoms totalled four or more on a given day. As soon as possible, but within $48 \mathrm{~h}$ after the call, a research nurse visited the child, took a throat swab for the detection of viruses and performed spirometry. This procedure was adopted from JOHNSTON et al. [8]. One week later, nurses administered a PAQLQ. The authors defined these weeks, starting with a predefined severity level for respiratory symptoms as "weeks of illness".

\section{Spirometry}

A handheld spirometer (Micro DL Micro Medical Systems, Rochester, UK) was used, standardised in accordance with the recommendations of the American Thoracic Society, and measured forced expiratory volume in one second (FEV1), forced vital capacity (FVC), maximum expiratory flow at $25 \%$ of FVC (MEF25) and maximum expiratory flow at $50 \%$ of FVC (MEF50). On each occasion spirometry was performed three times and the best result was taken. Spirometric data were obtained at inclusion, at the same moment as the baseline PAQLQ, and at the start of a week of illness. When children had multiple weeks of illness the mean spirometric values were calculated. Values for FEV1 and FVC were expressed as percentage of predicted values according to age, sex and height following the procedure proposed by QUANJER et al. [9] and for MEF25 and MEF50 as proposed by ROSENTHAL et al. [10].

\section{Virological assessments}

Throat swabs were cultured and searched for the presence of influenza virus A and B as described earlier [3] and the same method was applied for detecting parainfluenza 1,2 and 3 , adenovirus and respiratory syncytial virus. They were subsequently analysed by immunofluorescence for respiratory viruses using specific reagents (Dako, Glostrup, Denmark). Throat swabs were also tested by PCR for influenza A and B [11] and rhinoviruses [12].

\section{Randomisation and blinding}

Randomisation, packing and labelling took place by the manufacturer of the vaccine. All those involved, i.e. patients and parents, GPs and investigators, were blinded.

\section{Statistical methods}

The data were analysed by intention-to-treat. Differences between groups were analysed by Fisher's exact test in case of proportions and by ANOVA for continuous data.

\section{Results}

A total of 200 GPs in 144 practices participated. GPs selected 3,220 children and informed them and their parents by letter about the objectives of the study. In total, 1,365 children were willing to participate. After inclusion and informed consent, 696 children participated. Of these, 347 were vaccinated with influenza vaccine and 349 with placebo. In each group 344 participants provided diary data. The current authors have already described this trial profile in detail [3]. In the two seasons the study was conducted 
(1999-2000 and 2000-2001), the influenza vaccine strains matched well with epidemic virus strains $[13,14]$.

\section{Characteristics at inclusion and at baseline}

The groups were similar for characteristics at inclusion and baseline, except for history of allergy (table 1). Of all children, $55 \%$ had previously been vaccinated against influenza.

\section{Outcomes}

Weeks of illness. Altogether, 486 telephone calls were received reporting a score of four points or more concerning 347 children of which 178 children were in the vaccine group and 169 children were in the placebo group. In the vaccine group 117 children reported once, 40 twice, nine three, two four and one five time(s). In the placebo group 123 children reported once, 43 twice, nine three, two four and one five time(s). For 342 of these 347 children one or more outcomes during weeks of illness were available, 176 in the vaccine group and 166 in the placebo group. There were 43 weeks of illness in which influenza virus was detected (one double infection with influenza A and B), 22 in the vaccine group and 21 in the placebo group. Of those influenza-positive weeks, data on QoL were available in 40 cases and on symptoms and spirometry in 42 cases.

Quality of life. A baseline PAQLQ was obtained from 662 children, 331 in the placebo group and 331 in the vaccine group. For all children baseline scores both for the total score and the domain scores did not differ between the groups.

Table 1.-Baseline characteristics of the study population

\begin{tabular}{|c|c|c|}
\hline & Vaccine & Placebo \\
\hline At inclusion visits $n$ & 347 & 349 \\
\hline Age yrs & $10.5 \pm 3.2$ & $10.6 \pm 3.3$ \\
\hline Females $\%$ & 48.1 & 43.6 \\
\hline Age of onset of asthma yrs & $3.5 \pm 3.8$ & $3.3 \pm 3.4$ \\
\hline Ever vaccinated for influenza \% & 53.6 & 56.7 \\
\hline History of allergy $\%$ & 73.5 & 66.1 \\
\hline Child born in the Netherlands \% & 97.7 & 98.9 \\
\hline FEV1 \% predicted & $89.5 \pm 16.1$ & $88.9 \pm 16.0$ \\
\hline \multicolumn{3}{|l|}{ At baseline visits } \\
\hline Quality of life $n$ & 331 & 331 \\
\hline Total score & 6.16 & 6.16 \\
\hline Activities & 5.60 & 5.60 \\
\hline Emotions & 6.63 & 6.63 \\
\hline Symptoms & 6.05 & 6.05 \\
\hline Spirometry $n$ & 332 & 337 \\
\hline \multicolumn{3}{|l|}{ FEV1 } \\
\hline Mean & $2.13 \pm 0.76$ & $2.13 \pm 0.80$ \\
\hline$\%$ predicted & $93 \pm 14$ & $92 \pm 13$ \\
\hline \multicolumn{3}{|l|}{ FVC } \\
\hline Mean & $2.43 \pm 0.88$ & $2.43 \pm 0.96$ \\
\hline$\%$ predicted & $92 \pm 14$ & $90 \pm 14$ \\
\hline \multicolumn{3}{|l|}{ MEF50 } \\
\hline Mean & $2.68 \pm 1.10$ & $2.66 \pm 1.0$ \\
\hline$\%$ predicted & $90 \pm 25$ & $90 \pm 24$ \\
\hline \multicolumn{3}{|l|}{ MEF25 } \\
\hline Mean & $1.42 \pm 0.71$ & $1.42 \pm 0.67$ \\
\hline$\%$ predicted & $94 \pm 36$ & $96 \pm 36$ \\
\hline
\end{tabular}

Data are presented as mean \pm SD unless otherwise stated. FEV1: forced expiratory volume in one second; FVC: forced vital capacity; MEF50: Maximum expiratory flow at 50\% FVC; MEF25: maximum expiratory flow at $25 \%$ of FVC. ${ }^{\circ}$ : mean of scores ranging from $1-7$, with higher scores indicating better quality of life.
In 333 children a PAQLQ for a baseline week and a PAQLQ during one or more illness weeks was recorded. In children who reported weeks of illness no differences in baseline were found between the vaccine group and the placebo group.

Differences between baseline PAQLQ scores and scores during influenza-positive weeks of illness favoured the vaccine group, as did differences in the symptoms and activities domains (table 2), whilst there were no differences between baseline scores and scores in weeks of illness, irrespective of their cause. The proportion of children reaching the minimal important difference for the symptoms domain in influenzapositive weeks as well as in all weeks of illness were both significantly lowered in the vaccine group (table 2 ).

Symptom scores during weeks of illness. No differences were found between study arms for mean total or separate URT or LRT symptom scores and for symptom scores in influenzarelated weeks or all weeks of illness, irrespective of cause (table 3).

Spirometry. At inclusion spirometric data was acquired from 682 children and at baseline spirometric data was acquired from 669 children, 332 in the vaccine group and 337 in the placebo group (table 1). Both baseline spirometry and spirometry during the weeks of illness were available for 455 of the 486 weeks of illness; in the remaining 31 cases either one or both spirometric values were not available due to technical problems. No differences were found between vaccine and placebo for baseline spirometric values (table 1). At the onset of influenza-positive periods a significant difference in FVC percentage predicted favouring the vaccine group was found (table 4), with a nonsignificant trend for improvement of FEV1.

Daily symptom scores throughout the season. No differences were found for mean total daily scores on URT and LRT symptoms throughout the influenza season between the vaccine group and the placebo group (table 5) or for the number of days not fit to go to school or work because of those symptoms.

Detection of viruses. In 484 of 486 weeks of illness a throat swab was taken, one child on two occasions did not allow a throat swab to be taken. Viruses were detected in $136(28.1 \%)$ of 484 throat swabs (four double infections), 41 times influenza A $(8.5 \%)$, three times influenza B $(0.6 \%), 14$ times corona $(2.9 \%), 57$ times rhinovirus $(11.8 \%), 12$ times respiratory syncytial virus $(2.5 \%)$ and nine times miscellaneous viruses $(1.9 \%)$ (table 6). Detection rates were $37 \%$ for the vaccine group and 34\% for the placebo group for 1999-2000, and 19\% versus $26 \%$ for $2000-2001$, respectively. There were no significant differences in total detection rates or for separate viruses between the vaccine group and the placebo group.

\section{Discussion}

Influenza vaccination, compared with placebo, were found to improve health-related QoL in asthmatic children in the weeks of illness in which influenza virus was detected in their throat swabs. This improvement was found for overall asthma-related QoL as measured by the PAQLQ, as well as for its symptoms and activities domains. The findings for the symptoms domain concur with the findings using the minimal important difference as an additional parameter, relevant for clinical practice. In illness weeks a difference in FVC as per cent predicted favouring the vaccine group was also found. 
Table 2. - Mean quality of life (QoL) assessed by the Paediatric Asthma Quality of Life Questionnaire

\begin{tabular}{|c|c|c|c|c|c|c|}
\hline \multirow[t]{2}{*}{ QoL scores ${ }^{\#}$} & \multicolumn{3}{|c|}{ All weeks of illness } & \multicolumn{3}{|c|}{ Influenza-positive weeks } \\
\hline & Vaccine & Placebo & p-value & Vaccine & Placebo & p-value \\
\hline Scores $\mathrm{n}$ & 173 & 160 & & 21 & 19 & \\
\hline \multicolumn{7}{|l|}{ Mean } \\
\hline Total & 5.54 & 5.43 & 0.35 & 5.87 & 5.47 & 0.15 \\
\hline Activities & 4.85 & 4.73 & 0.38 & 5.23 & 4.82 & 0.27 \\
\hline Emotions & 6.30 & 6.26 & 0.28 & 6.47 & 6.38 & 0.75 \\
\hline Symptoms & 5.24 & 5.10 & 0.72 & 5.69 & 5.03 & 0.08 \\
\hline \multicolumn{7}{|c|}{ Mean differences between baseline } \\
\hline Total & -0.65 & -0.76 & 0.19 & -0.40 & -1.00 & 0.02 \\
\hline Activities & -0.82 & -1.00 & 0.14 & -0.49 & -1.31 & 0.02 \\
\hline Emotions & -0.35 & -0.40 & 0.56 & -0.21 & -0.41 & 0.29 \\
\hline Symptoms & -0.81 & -0.95 & 0.20 & -0.52 & -1.35 & 0.04 \\
\hline \multicolumn{7}{|c|}{ MID between baseline ${ }^{+}$} \\
\hline Total $\%$ & 50 & 61 & 0.06 & 48 & 68 & 0.22 \\
\hline Activities \% & 54 & 64 & 0.06 & 48 & 79 & 0.06 \\
\hline Emotions \% & 27 & 26 & 1.00 & 24 & 26 & 1.00 \\
\hline Symptoms \% & 52 & 65 & 0.01 & 43 & 79 & 0.03 \\
\hline
\end{tabular}

MID: minimal important difference. ${ }^{\#}$ : scores range 1-7, with higher scores indicating better QoL; ${ }^{\top}$ : negative differences, indicating a poorer QoL during weeks of illness; ${ }^{+}$: proportion of patients with a negative difference of $\geqslant 0.5$ points.

Table 3. - Mean respiratory tract symptom scores per day in children during weeks of illness

\begin{tabular}{|c|c|c|c|c|c|c|}
\hline & \multicolumn{3}{|c|}{ All weeks of illness } & \multicolumn{3}{|c|}{ Influenza-positive weeks } \\
\hline & Vaccine & Placebo & p-value & Vaccine & Placebo & p-value \\
\hline Outcomes n & 176 & 166 & & 22 & 20 & \\
\hline \multicolumn{7}{|l|}{ Upper respiratory tract } \\
\hline Runny or stuffy nose & 0.91 & 0.85 & 0.43 & 0.97 & 0.85 & 0.60 \\
\hline Sneezing & 0.33 & 0.29 & 0.44 & 0.44 & 0.38 & 0.60 \\
\hline Burning or watery eyes & 0.13 & 0.15 & 0.74 & 0.26 & 0.20 & 0.68 \\
\hline Sore throat & 0.38 & 0.35 & 0.52 & 0.32 & 0.45 & 0.24 \\
\hline Hoarseness & 0.12 & 0.12 & 0.98 & 0.06 & 0.15 & 0.21 \\
\hline Fever or shivering & 0.22 & 0.21 & 0.86 & 0.41 & 0.43 & 0.82 \\
\hline Headache & 0.39 & 0.33 & 0.30 & 0.37 & 0.54 & 0.27 \\
\hline Myalgia & 0.07 & 0.07 & 0.89 & 0.07 & 0.10 & 0.67 \\
\hline Total symptom score & 2.56 & 2.37 & 0.37 & 2.91 & 3.13 & 0.69 \\
\hline \multicolumn{7}{|l|}{ Lower respiratory tract } \\
\hline Cough during the day & 0.93 & 1.03 & 0.26 & 1.05 & 1.27 & 0.39 \\
\hline Cough during the night & 0.50 & 0.52 & 0.78 & 0.67 & 0.71 & 0.86 \\
\hline Wheeze during the day & 0.13 & 0.16 & 0.50 & 0.15 & 0.18 & 0.77 \\
\hline Wheeze during the night & 0.10 & 0.11 & 0.58 & 0.07 & 0.11 & 0.50 \\
\hline Difficult breathing or shortness of breath & 0.39 & 0.42 & 0.56 & 0.32 & 0.64 & 0.06 \\
\hline Total symptom score & 2.05 & 2.24 & 0.33 & 2.25 & 2.91 & 0.24 \\
\hline
\end{tabular}

Each separate score ranged from 0 (absent) to 3 (severe).

However, in influenza-positive weeks of illness no differences were found between the vaccine group and the placebo group for mean URT or LRT symptoms. Regarding all weeks of illness irrespective of their cause, no differences were found for mean URT or LRT symptoms or for spirometric parameters. With regard to respiratory symptoms throughout the seasons, influenza vaccination did not reduce URT and LRT symptoms in asthmatic children.

That influenza vaccination in this analysis improves asthmarelated QoL in weeks of illness is in contrast to previous findings of the authors on influenza-related asthma exacerbations. Earlier analysis showed no difference in the number, duration and severity of influenza-related asthma exacerbations as a result of vaccination [3].

How can this discrepancy be explained? First, in the authors' previous paper they defined exacerbations as episodes of LRT symptoms of $\geqslant 2$ days with symptom scores above the median for that child preceded by at least 1 day at or below the median and followed by at least 2 days at or below the median. In this way less severe and supplemental symptomatology may have been ignored and hence a possible effect might have escaped attention. Secondly, the duration of the exacerbations may not exactly cover the weeks of illness in which QoL was measured. Thirdly, the sensitivity of the PAQLQ for vaccine-related benefits may be higher than the diary symptom score list used by the authors [14]. This might well be the case, as questions on well-being, the use of more domains for measuring asthma, the greater scale range of the scores and the way of conducting the questionnaires by research nurses may detect changes that the simpler diary cards could not pick up.

The findings of no difference in the number of days with symptoms and the mean severity of symptoms throughout the seasons between study arms seem contradictory to the results 
Table 4.-Data on spirometric parameters

\begin{tabular}{|c|c|c|c|c|c|c|}
\hline & \multicolumn{3}{|c|}{ All weeks of illness } & \multicolumn{3}{|c|}{ Influenza-positive weeks } \\
\hline & Vaccine & Placebo & p-value & Vaccine & Placebo & $\mathrm{p}$-value \\
\hline Outcomes $\mathrm{n}$ & 172 & 157 & & 21 & 20 & \\
\hline FEV1 & $1.95 \pm 0.75$ & $2.00 \pm 0.86$ & 0.63 & $1.77 \pm 0.82$ & $1.59 \pm 0.67$ & 0.31 \\
\hline$\%$ predicted & $87 \pm 17$ & $85 \pm 17$ & 0.24 & $89 \pm 22$ & $80 \pm 20$ & 0.09 \\
\hline FVC & $2.25 \pm 0.85$ & $2.31 \pm 1.02$ & 0.60 & $2.06 \pm 0.52$ & $1.92 \pm 0.82$ & 0.53 \\
\hline$\%$ predicted & $87 \pm 16$ & $85 \pm 17$ & 0.14 & $91 \pm 18$ & $80 \pm 19$ & 0.08 \\
\hline MEF50 & $2.36 \pm 1.0$ & $2.38 \pm 1.0$ & 0.87 & $2.08 \pm 0.50$ & $1.83 \pm 1.0$ & 0.32 \\
\hline$\%$ predicted & $81 \pm 25$ & $79 \pm 24$ & 0.69 & $78 \pm 17$ & $75 \pm 29$ & 0.66 \\
\hline MEF25 & $1.29 \pm 0.64$ & $1.33 \pm 0.71$ & 0.56 & $1.11 \pm 0.28$ & $0.98 \pm 0.56$ & 0.35 \\
\hline$\%$ predicted & $88 \pm 36$ & $88 \pm 35$ & 0.86 & $83 \pm 21$ & $82 \pm 33$ & 0.92 \\
\hline Differences $^{\#} \mathrm{n}$ & 162 & 153 & & 21 & 20 & \\
\hline FEV1 & $-7 \pm 13$ & $-8 \pm 12$ & 0.31 & $-4 \pm 12$ & $-12 \pm 11$ & 0.06 \\
\hline FVC & $-5 \pm 14$ & $-6 \pm 14$ & 0.34 & $-0 \pm 12$ & $-11 \pm 15$ & 0.01 \\
\hline MEF50 & $-10 \pm 16$ & $-11 \pm 20$ & 0.59 & $-11 \pm 15$ & $-17 \pm 15$ & 0.17 \\
\hline $\mathrm{MEF} 25$ & $-8 \pm 29$ & $-10 \pm 30$ & 0.37 & $-13 \pm 22$ & $-13 \pm 19$ & 0.88 \\
\hline
\end{tabular}

Data are presented as mean \pm SD. FEV1: forced expiratory volume in one second; FVC: forced vital capacity; MEF50: maximum expiratory flow (MIF) at $50 \%$ of FVC; MEF 25: MIF at $25 \%$ of FVC. ${ }^{\#}$ : differences in $\%$ predicted comparing spirometry at baseline and during illness weeks.

Table 5.-Mean respiratory tract symptom scores per day throughout the influenza season

\begin{tabular}{|c|c|c|c|}
\hline & Vaccine & Placebo & p-value \\
\hline Scores $n$ & 344 & 344 & \\
\hline \multicolumn{4}{|l|}{ Upper respiratory tract } \\
\hline Runny or stuffy nose & 0.30 & 0.26 & 0.11 \\
\hline Sneezing & 0.12 & 0.10 & 0.29 \\
\hline Burning or watery eyes & 0.04 & 0.03 & 0.73 \\
\hline Sore throat & 0.07 & 0.06 & 0.10 \\
\hline Hoarseness & 0.03 & 0.03 & 0.64 \\
\hline Fever or shivering & 0.03 & 0.03 & 0.85 \\
\hline Headache & 0.10 & 0.09 & 0.41 \\
\hline Myalgia & 0.04 & 0.02 & 0.08 \\
\hline Total symptom score & 0.75 & 0.65 & 0.13 \\
\hline \multicolumn{4}{|l|}{ Lower respiratory tract } \\
\hline Cough during the day & 0.25 & 0.23 & 0.61 \\
\hline Cough during the night & 0.11 & 0.11 & 0.98 \\
\hline Wheeze during the day & 0.04 & 0.04 & 0.85 \\
\hline Wheeze during the night & 0.03 & 0.04 & 0.39 \\
\hline Difficult breathing or shortness of breath & 0.13 & 0.14 & 0.90 \\
\hline Total symptom score & 0.58 & 0.58 & 1.00 \\
\hline
\end{tabular}

Each separate score ranged from 0 (absent) to 3 (severe).

Table 6. - Pathogens detected by either culture, immunofluorescence or PCR in throat swabs in reported periods

\begin{tabular}{|c|c|c|c|c|c|}
\hline & Placebo 1999-2000 & Vaccine 1999-2000 & Placebo 2000-2001 & Vaccine $2000-2001$ & Total \\
\hline Influenza A-H3N2 & 6 & 2 & 0 & 0 & 8 \\
\hline Influenza A-H1N1 & 0 & 0 & 13 & 20 & 33 \\
\hline Influenza B & 0 & 0 & 2 & 1 & 3 \\
\hline Rhinovirus & 20 & 24 & 5 & 8 & 57 \\
\hline Coronavirus & 9 & 2 & 0 & 3 & 14 \\
\hline RSV & 4 & 7 & 0 & 1 & 12 \\
\hline Adenovirus & 1 & 0 & 1 & 2 & 4 \\
\hline Miscellaneous & 3 & 2 & 3 & 1 & 9 \\
\hline No virus found & 70 & 74 & 101 & 103 & 348 \\
\hline Reported periods & 111 & 112 & 124 & 139 & $486^{\#}$ \\
\hline
\end{tabular}

Data are presented as n. RSV: respiratory syncytial virus. ${ }^{\#}$ : column does not add up to 486 because of four double infections and two absent throat swabs.

of the authors' first study that vaccination reduced the length of all asthma exacerbations, irrespective of their cause. A possible explanation is that the use of a threshold symptom score before telephoning may have led to an under-detection of influenza and influenza-related asthma exacerbations. However, when all days with symptoms throughout the seasons are considered, instead of restricting it to asthma exacerbations, differences disappear. 
In the authors' study baseline scores of the PAQLQ were relatively high, implying a better QoL compared with other studies [15-17]; these latter studies were, however, performed in hospital-based populations. Children in the study probably had less severe asthma, because they were recruited via the GP. Weeks of illness were found to be characterised by a reduction in the total PAQLQ score of 0.65 in the vaccine group and 0.76 in the placebo group, as compared with baseline. This reduction compares well with the decrease in symptoms of 0.65 points in children with mild and severe asthmatic episodes, which has been reported before [15]. In the present study, compared with placebo, influenza vaccination improved the total score on QoL in influenza-positive periods by 0.6 points, which may be a meaningful improvement when considering the threshold for clinical relevance of 0.5 points for the MID. Other studies, also using the PAQLQ, found pre- and post-treatment improvements in total scores of 0.69 [16] and 0.25 [17] points. The PAQLQ has been reported to correlate with symptom scores and degree of asthma [15, 18, 19].

With regard to spirometry a lower FVC was found in influenza-positive periods in the placebo group compared with the vaccine group. The fall in FVC could be a result of influenza infection, but may have been caused by inadequate spirometry performance of the children whilst being ill. This is supported by the fact that no difference was found on other spirometric indices. In weeks of illness irrespective of their cause, no significant differences were found between study arms in percentage of change in spirometry parameters, as an indicator of relevant airway obstruction in asthma.

Using throat swabs, viruses were detected in $28.7 \%$ of the weeks of illness. Considering the viruses detected it is unlikely that an imbalanced distribution of viral infections confounded the results. Lower [20, 21] as well as higher [14, 22] rates of detection have been reported in asthmatic children. The differences in detection rate between studies can be explained by differences in sample techniques, laboratory techniques, time frames used and natural fluctuations in the viruses that cause infection.

Rhinovirus played the most important role in all these studies [14, 20-22], and were found in respectively 42, 46, 65 and $66 \%$ of cases. In the authors' study, rhinovirus was found in $41 \%$ of positive swabs. The incidence of influenza A and B varies with year and place. Of the 696 children in the authors' study, $43(8.9 \%)$ had influenza detected by culture or PCR. In the above-mentioned studies $[14,20-22]$ influenza was detected in respectively $1,6.7,7.4$ and $19.4 \%$ of participating children. In a recent study, TSAI et al. [23] examined the relationship between clinical symptoms and respiratory infection using conventional laboratory methods in paediatric outpatients during 3 yrs $(1997,1998,1999)$. In 35\% of these cases one or more viruses were detected; influenza A was isolated in $5.5 \%$ and $\mathrm{B}$ in $2.6 \%$ of cases with acute respiratory tract conditions. In the authors' study only small differences in the number and type of viral infections between vaccine group and placebo group were found.

What is the clinical relevance of the present findings? Vaccination rates in children with asthma are reported to be very low $[24,25]$. One of the reasons why physicians do not recommend influenza vaccination is disbelief in the positive effects of vaccination [26]. Among patients, reasons not to obtain influenza vaccination are doubts about the efficacy of influenza vaccine, fear of possible side-effects, and nonrecommendation by their doctor $[27,28]$. As far as is known by the authors this is the first randomised placebo-controlled study to demonstrate beneficial effects of influenza vaccination in children with asthma. In the current study influenza vaccination, compared with placebo, was associated with a relevant improvement in the QoL of asthmatic children in the weeks of illness in which influenza virus was detected. However, the current study had relatively low influenzapositive weeks of illness and could not detect any favourable effect of influenza vaccination on influenza-related asthma exacerbations and respiratory symptoms throughout the season. Hence, the question remains whether or not it is worth the effort to promote vaccination of all asthmatic children aged 6-18 yrs in general practice.

Therefore, to justify routine influenza vaccination in asthmatic children, the authors strongly recommend that further randomised placebo-controlled trials be carried out to assess the clinical effectiveness as well as the cost-effectiveness of influenza vaccination in children with asthma.

\begin{abstract}
Acknowledgements. The authors thank P. Rothbarth for challenging them to design the study. They also thank the children, parents, and general practitioners who took part in the study, as well as the research nurses and L. Euser, who coordinated the fieldwork.
\end{abstract}

\section{References}

1. Nicholson KG, Snacken R, Palache AM. Influenza immunization policies in Europe and the United States. Vaccine 1995; 13: 365-369.

2. Cates CJ, Jefferson TO, Bara AI, et al. Vaccines for preventing influenza in people with asthma (Cochrane Review). Oxford: Update Software; 2002.

3. Bueving HJ, Bernsen RM, De Jongste JC, et al. Influenza vaccination in children with asthma: randomized doubleblind placebo-controlled trial. Am J Respir Crit Care Med 2004; 169: 488-493.

4. Juniper EF. How important is quality of life in pediatric asthma? Pediatr Pulmonol Suppl 1997; 15: 17-21.

5. Neuzil KM, Hohlbein C, Zhu Y. Illness among schoolchildren during influenza season: effect on school absenteeism, parental absenteeism from work, and secondary illness in families. Arch Pediatr Adolesc Med 2002; 156: 986-991.

6. Juniper EF, Guyatt GH, Feeny DH, et al. Measuring quality of life in children with asthma. Qual Life Res 1996; 5: 35-46.

7. Raat H, Bueving HJ, De Jongste JC, et al. Responsiveness, longitudinal- and cross sectional construct validity of the Pediatric Asthma Quality of Life Questionnaire (PAQLQ) in Dutch children with asthma. Qual Life Res 2004; (In press).

8. Johnston SL, Pattemore PK, Sanderson G, et al. Community study of role of viral infections in exacerbations of asthma in 9-11 year old children. BMJ 1995; 310: 1225-1229.

9. Quanjer PH, Borsboom GJ, Brunekreef B, et al. Spirometric reference values for white European children and adolescents: Polgar revisited. Pediatr Pulmonol 1995; 19: 135-142.

10. Rosenthal M, Bain SH, Cramer D, et al. Lung function in white children aged 4 to 19 years: I Spirometry. Thorax 1993; 48: 794-802.

11. Fouchier RA, Bestebroer TM, Herfst S, et al. Detection of influenza A viruses from different species by PCR amplification of conserved sequences in the matrix gene. $J$ Clin Microbiol 2000; 38: 4096-4101.

12. Pitkaranta A, Arruda E, Malmberg H, et al. Detection of rhinovirus in sinus brushings of patients with acute community-acquired sinusitis by reverse transcription-PCR. J Clin Microbiol 1997; 35: 1791-1793.

13. Nieuwsbrief influenza-surveillance [Newsletter on influenza Surveillance] 1999/2000; 8(Pt 8).

14. Nieuwsbrief influenza-surveillance [Newsletter on influenza Surveillance] 2000/01; 9(Pt 5).

15. Reichenberg K, Broberg AG. Quality of life in childhood asthma: use of the Paediatric Asthma Quality of Life 
Questionnaire in a Swedish sample of children 7 to 9 years old. Acta Paediatr 2000; 89: 989-995.

16. Knorr B, Matz J, Bernstein JA, et al. Montelukast for chronic asthma in 6- to 14-year-old children: a randomized, double-blind trial. Pediatric Montelukast Study Group. JAMA 1998; 279: 1181-1186.

17. Lemanske RF Jr, Nayak A, McAlary M, et al. Omalizumab improves asthma-related quality of life in children with allergic asthma. Pediatrics 2002; 110: e55.

18. Tauler E, Vilagut G, Grau G, et al. The Spanish version of the paediatric asthma quality of life questionnaire (PAQLQ): metric characteristics and equivalence with the original version. Qual Life Res 2001; 10: 81-91.

19. Clarke E, Sulaiman S, Chew Fook T, et al. Pediatric asthma quality of life questionnaire: validation in children from Singapore. Asian Pac J Allergy Immunol 1999; 17: 155161.

20. Mitchell I, Inglis JM, Simpson H. Viral infection as a precipitant of wheeze in children. Combined home and hospital study. Arch Dis Child 1978; 53: 106-111.

21. Horn ME, Brain EA, Gregg I, et al. Respiratory viral infection and wheezy bronchitis in childhood. Thorax 1979; 34: 23-28.
22. Mertsola J, Ziegler T, Ruuskanen O, et al. Recurrent wheezy bronchitis and viral respiratory infections. Arch Dis Child 1991; 66: 124-129.

23. Tsai HP, Kuo PH, Liu CC, et al. Respiratory viral infections among pediatric inpatients and outpatients in Taiwan from 1997 to 1999. J Clin Microbiol 2001; 39: 111-118.

24. Szilagyi PG, Rodewald LE. Missed opportunities for influenza vaccination among children with asthma. Pediatr Infect Dis $J$ 1992; 11: 705-708.

25. Kramarz P, DeStefano F, Gargiullo PM, et al. Influenza vaccination in children with asthma in health maintenance organizations. Vaccine Safety Datalink Team. Vaccine 2000; 18: 2288-2294.

26. Nichol KL, Zimmerman R. Generalist and subspecialist physicians' knowledge, attitudes, and practices regarding influenza and pneumococcal vaccinations for elderly and other high-risk patients: a nationwide survey. Arch Intern Med 2001; 161: 2702-2708.

27. Nichol KL, MacDonald R, Hauge M. Factors associated with influenza and pneumococcal vaccination behavior among high-risk adults. J Gen Intern Med 1996; 11: 673-677.

28. Fiebach NH, Viscoli CM. Patient acceptance of influenza vaccination. Am J Med 1991; 91: 393-400. 